\title{
Minimally Invasive Aortic Valve Replacement via Right Mini- thoracotomy versus Conventional Full Median Sternotomy: Tertiary Center Experience
}

\author{
Mohamed Alassal $^{1 *}$, Mohamed Saffan ${ }^{1}$, Basem Mofreh ${ }^{1}$, Samer AbdEl-Shafi ${ }^{2}$, Moataz Rezk ${ }^{1}$, Ehab Fawzy ${ }^{1}$, \\ Mohammed Obida ${ }^{1}$, Mahmoud Elemam ${ }^{1}$ and Yosry Shaheen ${ }^{1}$ \\ ${ }^{1}$ Cardiothoracic Surgery Department, Benha University, faculty of medicine, Egypt \\ ${ }^{2}$ Cardiothoracic Surgery Department, Military Medical Academy, Cairo, Egypt
}

Received: 20 December, 2017; Accepted: 15 January, 2018; Published: 22 January, 2018

*Corresponding author: Mohamed Alassal, Cardiothoracic Surgery Department, Benha University, faculty of medicine, Egypt. E-mail: dmohamedabdelwahab@gmail.com

\begin{abstract}
Introduction: Minimally invasive approach to Aortic Valve Replacement (AVR) is increasingly accepted as a valid alternative to full sternotomy approach, as to reduce operative trauma with the final aim to improve post-operative outcomes. The aim of this work is to evaluate the feasibility of minimally invasive aortic valve surgery through a right mini-thoracotomy, and hence to minimize the surgical access to achieve better cosmetic results, less postoperative discomfort and faster recovery while maintaining the same level of safety and favorable results as with conventional surgery.
\end{abstract}

Methods: In this study a 150 patients with Aortic Valve Disease (AVD) requiring aortic valve surgery were none randomly selected. The study was performed at Benha University Hospital \& the Armed Forces Hospitals. Seventy five patients underwent aortic valve surgery by traditional median sternotomy with central cannulation (group B), the other seventy five patients by right mini-thoracotomy on $2^{\text {nd }}$ or $3^{\text {rd }}$ right intercostal space with peripheral femoral cannulation (group A) Endpoints were overall postoperative complications, major adverse cardiac related complications, use of blood products and need for transfusions, bypass time and cross clamp time, ventilation time and length of hospital-stay.

Results: Minimally invasive AVR was associated with a significant reduction in need for blood and blood products transfusions, as well as postoperative cardiac and non-cardiac complications. Post-operative pain was significantly reduced in the mini-invasive group, a trend to lower mean ventilation times, ICU stay and hospital-stay in the miniinvasive group was also detected.

Conclusion: Minimally invasive aortic valve surgery has evolved into a well tolerated, efficient surgical treatment option in experienced centers, providing greater patient satisfaction and lower complication rates. Potential advantages of Minimally Invasive Aortic Valve Replacement (MIAVR) arise from the concept that patient morbidity and potential mortality could be reduced without compromising the excellent results of the conventional procedure and include improved cosmetic results, safer access in the case of re-operation, less postoperative bleeding, less blood transfusions, lower intensive care unit and in-hospital stays, as well as the absence of sternal wound infection.

\section{Introduction}

Full median sternotomy has been well established as a standard approach for all types of open heart surgery for many years. Although well established, the full sternotomy incision has been frequently criticized for its length, post-operative pain and possible complications like wound infection and instability [1].

Developments in minimally invasive aortic surgery began in the mid-1990s with the pioneering work of Cohn, Cosgrove, Navia and others. Technological advancements in instrumentation, assisted vision, and CPB support have followed closely and have expedited this evolutionary process. Within a few short years MIAVS have gone from simple modifications of conventional techniques to near totally endoscopic operations [2-5].

Because of the continuous trend towards less invasive procedures, cardiac operations have become increasingly more sophisticated and complex. Minimally invasive techniques in cardiac operations require higher surgical abilities to accomplish the same quality compared with the traditional procedures with Cardio-Pulmonary Bypass (CPB) or full sternotomy [6].

Minimally invasive aortic valve surgery has evolved into a well tolerated, efficient surgical treatment option in experienced centers, providing greater patient satisfaction and lower complication rates. Potential advantages of minimally invasive aortic valve replacement arise from the concept that patient morbidity and potential mortality could be reduced without compromising the excellent results of the conventional procedure and include improved cosmetic results, safer access in the case of re-operation, less post-operative bleeding, fewer blood transfusions, lower intensive care unit and in-hospital stays, as well as the absence of sternal wound infection [7].

The key to successful thoracic surgical procedures is adequate and proper exposure. A well chosen thoracic incision provides effortless and excellent exposure for almost any procedure. However, an ill chosen or an improperly placed or performed 
incision often leads to a difficult and frustrating procedure [8].

There is a learning curve associated with any cardiac surgical procedure, despite what experienced surgeons now consider routine and simple. In order to overcome the conceptual "learning curve", surgeons need to consider this the standard of care in isolated AVR surgery and make it reality. Once proficiency is acquired, the minimal access approach may be the procedure of choice for AVR [9].

The right mini-thoracotomy approach for aortic valve replacement was first described by Rao and Kumar and was reproduced by Galloway and others.

All patients scheduled for right mini-thoracotomy should undergo computed tomography scan without contrast enhancement to evaluate the anatomic relationship among the intercostal spaces, ascending aorta, and aortic valve. Patients are suitable for this approach only if the following criteria are met: (I) at the level of main pulmonary artery, the ascending aorta is rightward (more than one half located on the right in respect to the right sternal border); (II) the distance from the ascending aorta to the sternum does not exceed $10 \mathrm{~cm}$ (III) the $\alpha$ angle (angle between the angle midline and the inclination of ascending aorta) should be $>45$ (Figure $1 \mathrm{~A}$ and $1 \mathrm{~B}$ ) [10].

\section{Materials and Methods}

\section{Patient Selection}

In this retrospective study, a 150 patients with Aortic Valve Disease (AVD) requiring aortic valve surgery were none randomly selected. The study was performed in the period from January 2014 through June 2016 at Benha University Hospital \& the Armed Forces Hospitals (El Maadi, El Galaa and kobryElkobba). Seventy five patients underwent aortic valve surgery by traditional median sternotomy with central cannulation (group B), the other seventy five patients by right mini-thoracotomy on 2 nd or 3rd right intercostal space with peripheral femoral cannulation (group A).

- In group "A", age ranged from 21-71 years with a mean of $49.1 \pm 16$.

- While in group "B" age ranged from 24-73 years with a mean of $47.6 \pm 13$.

- In group "A", there was 45 males (60\%) and 30 females (40\%), while in group "B" there was 48 males $(66.6 \%)$ and 27 females $(33.3 \%)$.
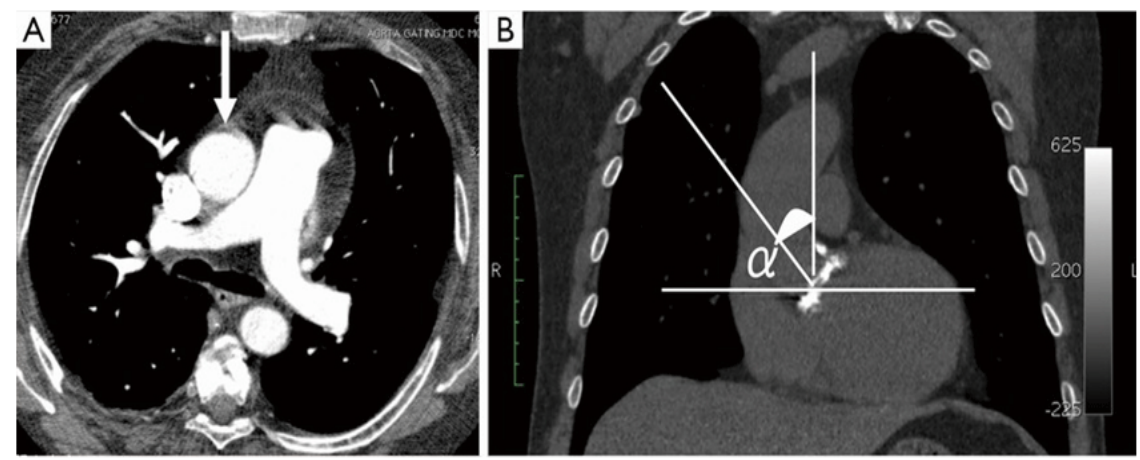

Figure 1: CT angiography (A\&B) showing the suitability of the patient for the minimally invasive approach

\section{Surgical Technique}

Anesthesia is provided according to the standard protocol used for conventional aortic valve surgery with the only exception for a double lumen endotracheal tube for intubation. Transesophageal echocardiography was used in all patients for monitoring the heart and valve functions throughout the operation. Two defibrillator pads are placed across the chest wall to guarantee effective electric conduction. The patient is placed in a supine position (Figure 2).

The procedure is carried out through a $5-6 \mathrm{~cm}$ skin incision beginning at the right sternal border extending to the right antero-lateral portion of the chest wall. After that the pectoralis muscle is opened by cautery followed by the intercostals muscle entering into the $2^{\text {nd }}$ or $3^{\text {rd }}$ ICS. We use a soft tissue retractor and rib retractor to obtain further exposure (Figure 3).

When both femoral arterial and venous cannulation are used, a transverse 3-4 $\mathrm{cm}$ incision along the inguinal fold over the pulsating femoral artery projection is made to expose the vessels. Purse string sutures with prolene 5/0 taken over the artery and vein. When heparin is administered, femoral artery and vein cannulation are performed utilizing a Seldinger technique. We perform arterial cannulation first; the cannula should never be forced and should advance easily. The cannula is then secured over the vessel with a tourniquet and connected to the CPB arterial line (Figure 4).

After initiation of the CPB, venous drainage is achieved with vacuum assistance of approximately $-40 \mathrm{mmHg}$. Currently, several options are available for aortic cross-clamping. An external Cosgrove flexible or a Chitwood clamp can be used (Figure 5).

After cross-clamping of the aorta and cardioplegia solution delivered through the aortic root, a transverse aortotomy is made approximately $1.5 \mathrm{~cm}$ above the take off the right coronary artery, slightly above the level of the sino-tubular ridge. The leaflets of 


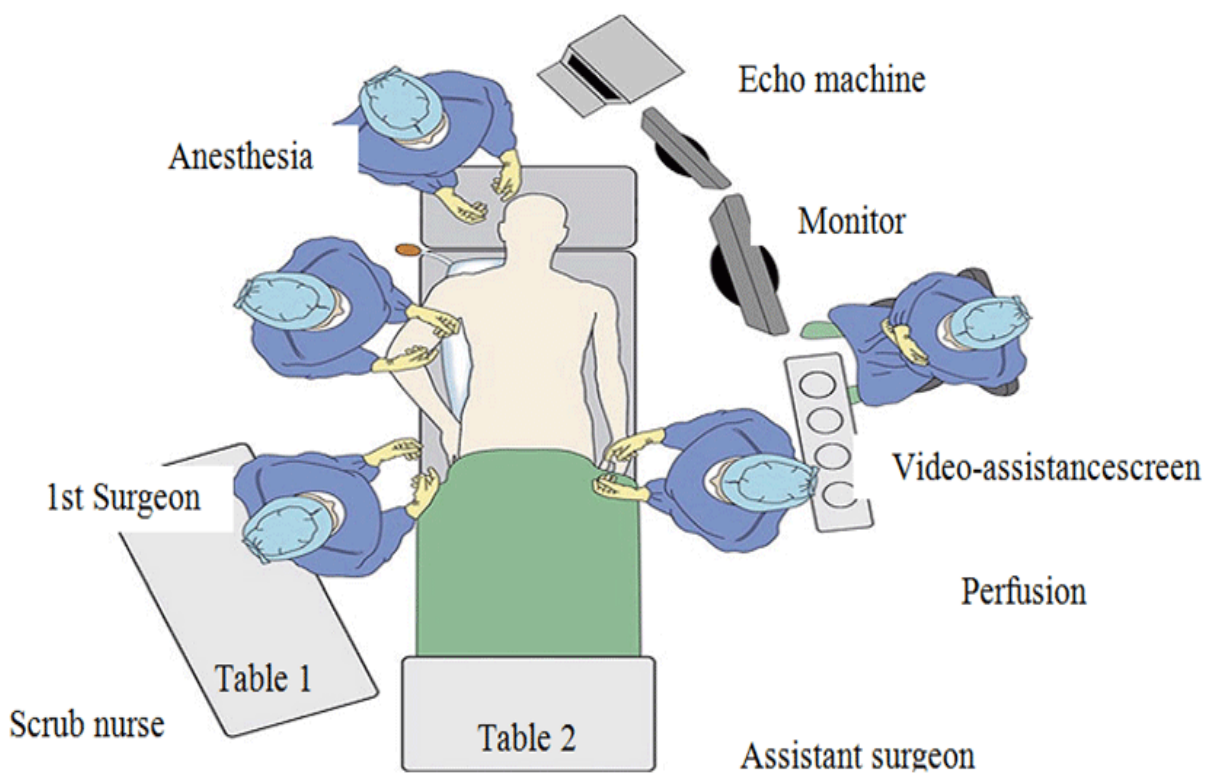

Figure 2: Positioning of the surgical team in the operating room (Adopted from Minimally Invasive Thoracic and Cardiac Surgery Textbook and Atlas, 2012).

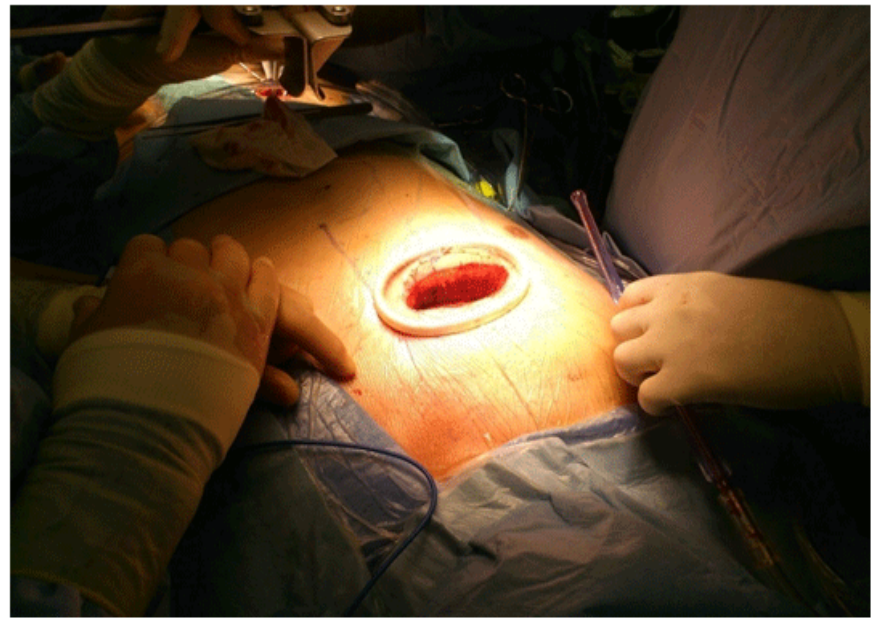

Figure 3: The use of soft tissue retractor

the aortic valve are excised to the level of the annulus and the annulus is thoroughly debrided of any calcium. Braided 2-0 sutures with pledgets are utilized. The annulus is encircled with interrupted mattress sutures extending from the ventricular to the aortic surface (inverting).

After placement, the suture bundles are divided into two equal portions and implanted in the sewing ring and the prosthesis seated. Once the operative procedure has been completed, rewarming of the patient is begun. Removal of air from the heart (de-airing) is accomplished through right superior pulmonary vein vent. However, supplementary de-airing is also performed through the aorta. Simultaneously, the surgeon gently massages the left ventricle so that entrapped air evacuates through the vent. The aortic clamp is removed. Full venous return is then allowed to drain into the cardiopulmonary circuit, and the heart is collapsed.

\section{Statistical Analysis}

Peri-operative data were statistically analyzed using Statistical Package Of Social Science (SPSS), EPIcalc software programs using the arithmetic mean, standard deviation using hypothesis student's " $t$ " tests for quantitative data analysis, while qualitative data (ordinal, categorical) were analyzed using The chi-square test $\left(\mathrm{x}^{2}\right)$ (Fisher's Exact Test).

Predicts for prolonged hospital stay, morbidities were studies using multivariate analysis.

For all statistical comparisons, a $\mathrm{P}$ value of $<0.05$ was considered significant and a $\mathrm{P}$ value of $<0.01$ was considered highly significant. 


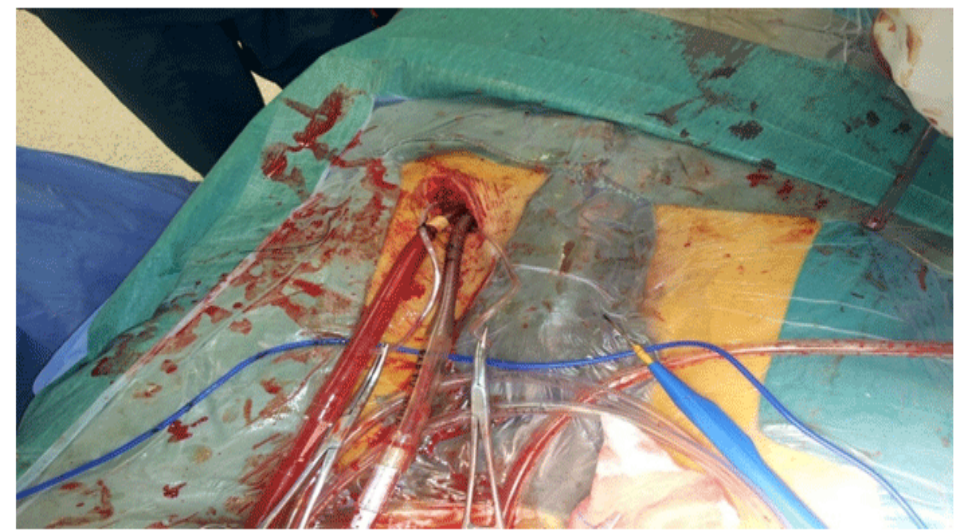

Figure 4: Cannulation of both femoral artery and vein

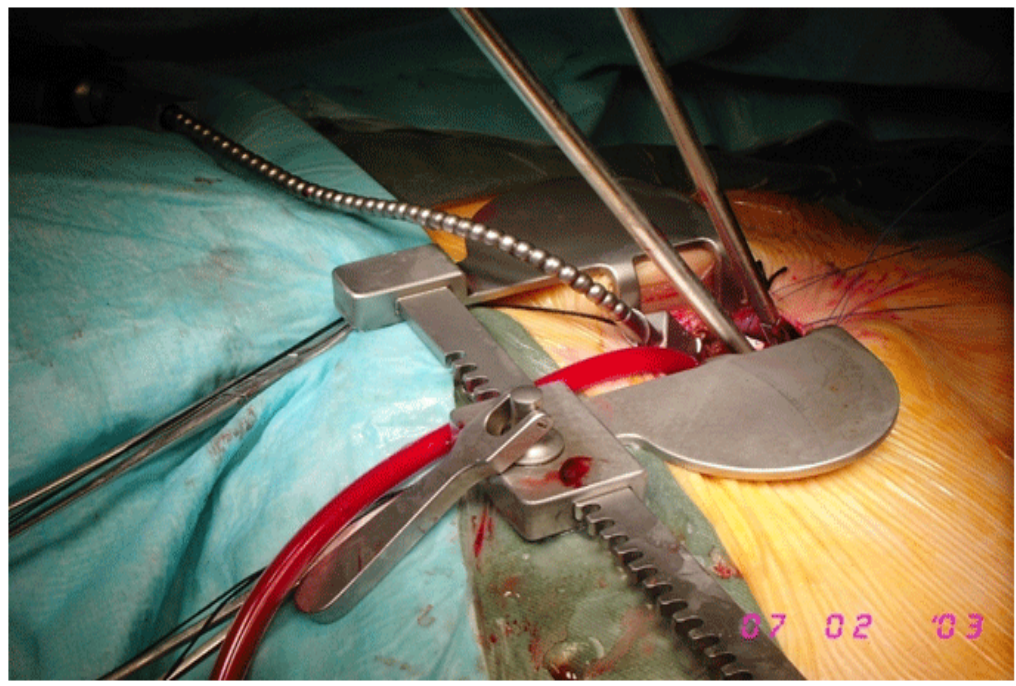

Figure 5: Use of flexible cross clamp

\section{Results}

This retrospective none randomized study compared the procedure and early postoperative outcome of the standard sternotomy approach versus the minimally invasive approach through right mini-thoracotomy by using direct vision. This study was conducted on 150 patients. All the patients completed the study without any mortality. The patients were classified into two groups: Group A: minimally invasive group. This group included 75 patients requiring aortic valve surgery and was approached through right mini-thoracotomy technique. Group B: Full sternotomy group. This group included 75 patients requiring aortic valve surgery and was approached through a conventional median sternotomy. There was highly statistical significance between the two groups as regards the cross clamp time and the total bypass time which is much shorter time in group B.

The length of the incision was compared in the two groups, The mean length of incision in group " $A$ " was $5.9 \pm 0.46 \mathrm{~cm}$ ranged from 5 to $8 \mathrm{~cm}$, while in group " $\mathrm{B}$ " the mean length was $21.4 \pm 1.5$ $\mathrm{cm}$ ranged from 18 to $23 \mathrm{~cm}$ (Table 1 ).

In group "A" all cases done through right mini-thoracotomy without the need to be converted to median sternotomy.

The ventilation time for group "A" ranged from 2.5-7 hours, with a mean $4.16 \pm 0.9$ hours. In group " $\mathrm{B}$ " the ventilation time ranged from 4-9 hours with a mean $5.9 \pm 1.5$ hours (Table 2).

There is a highly statistically significant difference between the two groups as regards the blood drainage in the first 24 hours, In group "A", blood drainage ranged from $150-500 \mathrm{ml}$ during the first 24 hour, with a mean of $288.3 \pm 86.78 \mathrm{ml}$ / first 24 hour. In group "B", the blood loss ranged from $200-800 \mathrm{ml}$ during the first 24 hour, with a mean of $486.3 \pm 177.67 \mathrm{ml}$ / first 24 hour. ICU stay in the minimally invasive group is less than the sternotomy group, with statistically highly significant difference.

Post-operative pain was less in group (A) with highly statistically significant difference.

Post-operative pain score using the visual analogue scale was compared in the two groups starting on day one after extubation till the $5^{\text {th }}$ postoperative day. In group (A) the mean pain score in the first post-operative day post extubation was $7.6 \pm 0.49$. This score decreased in the second post-operative day to $5.8 \pm$ 
Table 1: Length of skin incision in both groups

\begin{tabular}{|l|l|l|l|l|}
\hline & Group A & Group B & P value & Sig. \\
\hline Range (cm) & $5-8$ & $18-23$ & & \\
\hline Mean \pm SD (cm) & $5.9 \pm 0.46$ & $21.4 \pm 1.1$ & $<0.01$ & HS \\
\hline
\end{tabular}

Table 2: Ventilation, blood loss, blood transfusion and total ICU stay

\begin{tabular}{|c|c|c|c|c|}
\hline & Group A & Group B & $P$ value & Sig. \\
\hline \multicolumn{3}{|l|}{ Ventilation (hours) } & \multirow{4}{*}{$<0.01$} & \multirow{4}{*}{ HS } \\
\hline Range & $2.5-7$ & $4-9$ & & \\
\hline Mean & 4.16 & 5.9 & & \\
\hline SD & 0.9 & 1.5 & & \\
\hline \multicolumn{3}{|l|}{ Blood loss (ml) } & \multirow{4}{*}{$<0.01$} & \multirow{4}{*}{ HS } \\
\hline Range & $150-500$ & $200-800$ & & \\
\hline Mean & 288.3 & 486.3 & & \\
\hline SD & 86.7 & 177.67 & & \\
\hline \multicolumn{3}{|c|}{ Blood transfusion (unit) } & \multirow{4}{*}{$<0.01$} & \multirow{4}{*}{ HS } \\
\hline Range & $0-3$ & $1-4$ & & \\
\hline Mean & 1.4 & 2.5 & & \\
\hline SD & 0.7 & 0.8 & & \\
\hline \multicolumn{3}{|l|}{ ICU stay (day) } & \multirow{4}{*}{$<0.01$} & \multirow{4}{*}{ HS } \\
\hline Range & $1-3$ & $2-4$ & & \\
\hline Mean & 1.56 & 2.4 & & \\
\hline SD & 0.53 & 0.6 & & \\
\hline $\begin{array}{l}\text { Re-exploration for } \\
\text { bleeding }\end{array}$ & $3(3.3 \%)$ & $0(0.0 \%)$ & & \\
\hline
\end{tabular}

0.69. Pain score in group (B) during the first 24 hours was $9.2 \pm$ 0.76 which decreased to $7.7 \pm 0.78$ in the second post- operative day. This data showed that pain was less in group (A) with highly statistically significant difference as shown in results (Table 3).

The total hospital stay in the minimally invasive group was less than sternotomy group, and this difference has a highly statistical significance (Table 4).

Wound satisfaction was comparable in the two groups which showed that 66 cases (90\%) of group (A) were satisfied about their wound scar after mini-thoracotomy incision which was very small compared to wound scar after full sternotomy, while only 9 cases $(10 \%)$ not satisfied about their femoral wound scar. But in group (B) there were 63 cases (83.3\%) not satisfied about their wound scar and only 12 cases $(16.6 \%)$ were satisfied about their wound scar (Table 5).

Table 3: Pain score among the two groups (mean \pm SD)

\begin{tabular}{|c|c|c|c|c|}
\hline & Group A & Group B & P value & Sig. \\
\hline 1ST Day post-operative & $7.6 \pm 0.49$ & $9.2 \pm 0.76$ & $<0.01$ & HS \\
\hline 2ND Day post-operative & $5.8 \pm 0.69$ & $7.7 \pm 0.78$ & $<0.01$ & HS \\
\hline 5th day post operative & $3.9 \pm 1.54$ & $7.5 \pm 1.47$ & $<0.01$ & HS \\
\hline
\end{tabular}

\begin{tabular}{|c|c|c|c|c|}
\hline Table 4: Total Hospital Stay in days \\
\hline & Group A & Group B & P value & Sig. \\
\hline Range & $5-8$ & $7-11$ & & \\
\cline { 1 - 3 } Mean & 6.6 & 8.8 & $<0.01$ & HS \\
\hline SD & 0.9 & 0.8 & & \\
\hline
\end{tabular}

Table 5: patient's satisfaction about their wound scar

\begin{tabular}{|c|c|c|c|c|}
\hline & GROUP A & GROUP B & P value & SIG \\
\hline Wound Satisfaction & $66(90 \%)$ & $12(16.6 \%)$ & $<0.01$ & HS \\
\hline
\end{tabular}

\section{Discussion}

Refinements in surgical techniques have reduced morbidity and mortality related to valve operations. Innovative, less invasive approaches for the surgical treatment of aortic valve disease were introduced with success. Minimally invasive aortic valve surgery has evolved into a well-tolerated, efficient surgical treatment option in experienced centers, providing greater patient satisfaction and lower complication rates. It almost safe as median sternotomy for primary aortic valve surgery and could be used as an initial approach to aortic valve surgery [11]. In the past decade, aortic valve surgery utilizing minimally invasive approaches has been employed in an effort to decrease the "invasiveness" of the procedure. It has been difficult to consistently demonstrate objective benefits to minimally invasive techniques for aortic valve replacement (mini invasiveAVR). Reduced pain and hospital length of stay, decreased time until return to full activity, and decreased blood product use have been demonstrated $[12,13]$. The patients having aortic valve replacement using the minimally invasive procedures, spent a shorter time in the intensive care unit and had less need for postoperative ventilator usage (both number of patients using a ventilator and the mean time of use) in comparison with patients undergoing conventional sternotomy. In our study the mean age in group "A" was $49.1 \pm 16.1$ years, while in group "B", it was 47.6 \pm 13.5 years so the age groups in our study are relatively younger than the age groups in other studies. Donald D. Glower, et al. reported a mean age of $67 \pm 14(20-90)$ years in thoracotomy group and $63 \pm 14(22-92)$ years in sternotomy group $[14,15]$. The length of the incision was compared in the two groups. The mean length of incision in group "A" was $5.9 \pm 0.46 \mathrm{~cm}$ ranged from 5 to $8 \mathrm{~cm}$. While in group "B" the mean length was $21.4 \pm 1.1$ $\mathrm{cm}$ ranged from 18 to $23 \mathrm{~cm}$ which is statistically higher than that of group "A" (P value < 0.01). Mauro Del Giglio, et al. reported that Aortic valve replacement was performed through a 4 to $6 \mathrm{~cm}$ skin incision at the third intercotal space. There was a highly statistical significance difference between the two groups as regards the cross clamp time and the total bypass time which is much shorter in group B. One of the dis-advantages of the mini-thoracotomy approach is that it needs a learning curve for the surgeon and the all team to be able to perform the procedure through a smaller incision in a faster time. Daniyar Gilmanov, et al. mentioned that that the median of total bypass time was 108 (87 to 137) and Cross clamp time was 75 (57 to 97). In mini-thoracotomy group all cases done through right mini-thoracotomy without the need to be converted to median sternotomy. Daniyar Gilmanov, et al. 
mentioned that $19(2.2 \%)$ patients were converted to median sternotomy. In other study as Mattiaglauber, et al. reported that $2(1.5 \%)$ patients were converted to median sternotomy. In our study $63(86.6 \%)$ patients were connected to bypass through cannulation of both femoral artery and vein, while 12 (13.3 $\%)$ patients were connected through direct aortic cannula and percutaneous femoral vein cannulation because of peripheral arterial disease or small femoral artery $[16,7,17]$. Donald D Glower, et al. reported that 15 patients (3.3\%) have femoral artery cannulation, 5 patients $(1.1 \%)$ had axillary artery cannulation, while 432 patients $(95.5 \%)$ had central aortic cannulation. In our study, the ventilation time was 3.5-5 hours, with a mean $4.16 \pm 0.9$ hours for group A. In group "B" the ventilation time ranged from 4-9 hours with a mean $5.9 \pm 1.5$ hours. This shows that there is a high statistically significant difference between the two groups. Mattiaglauber, et al. study mentioned that postoperative mechanical ventilation is lower in patients undergoing mini-thoracotomy procedure surgery which ranged from 5-9 hours, while it was 6-11 hours in sternotomy group [15,17]. One of the most important advantages of the less invasive technique is the lesser incidence of post-operative bleeding and lesser requirement for re-exploration. In group " $\mathrm{A}$ ", blood drainage ranged from $150-500 \mathrm{ml}$ during the first 24 hour, with a mean of $288.3 \pm 86.78 \mathrm{ml} /$ first 24 hour. In group "B", the blood loss ranged from $200-800 \mathrm{ml}$ during the first 24 hour, with a mean of $486.3 \pm 177.67 \mathrm{ml} /$ first 24 hour, this shows that there is a highly statistically significant difference between the two groups as regards the blood drainage in the first 24 hours.

Daniyar Gilmanov, et al. had a different look for bleeding and transfusion which is; did the patient have persistent bleeding or not? did this bleeding need for $2^{\text {nd }}$ look or exploration or not? And reported that was more important than reporting the bleeding amount, however; less bleeding may be the result of smaller incision, which lessens the potential for bleeding. It is possible to stop bleeding from a minimally invasive incision during entry, whereas sternal bleeding from a standard sternotomy continues throughout the operative procedure. 3 patients $(3.3 \%)$ in group "A" required re-exploration for bleeding through the thoracotomy inscision with no need to conversion to sternotomy, with no one in group "B". Mattiaglauber, et al. reported that the incidence of re-exploration after mini-thoracotomy group were 9 (6.5\%) patients, while in sternotomy group were $6(4.3 \%)$ patients $[9,17]$.

The total intensive care unit (ICU) stay was comparable in both groups. In group "A", the ICU stay ranged from 1-3 days, with a mean of $1.56 \pm 0.53$ days, while in group "B" the range was $2-4$ days with a mean of $2.4 \pm 0.6$ days, which shows that the ICU stay in the minimally invasive group is less than the sternotomy group, with statistically highly significant difference. Mauro Del Giglio, et al. reported that the ICU stay ranged from (38-59.5 hours) with a median of 44 hours. Most of the studies performed, showed that the mean ICU stay was less in the mini-thoracotomy group. Evaluation of post-operative pain by visual analogue pain scale was used in the study. In group (A) the mean pain score in the first post-operative day after extubation was $7.6 \pm 0.49$. This score decreased in the second post-operative day to $5.8 \pm 0.69$, Pain score in group (B) during the first 24 hours was $9.5 \pm 0.5$ which decreased to $7.8 \pm 0.8$ in the second post- operative day. After 3 month the pain score using the visual analogue scale was compared in the two groups. In group (A) the mean pain score was $1.66 \pm 0.47$. Pain score in group (B) was $3.46 \pm 0.46$ with highly statistically significance differencev. This data showed that pain was less in group (A) with highly statistically significant difference [16] Other studies (Alejandro Aris et al 1999) reported that pain levels decreased progressively during the first 7 days post-operatively, and they found that patients suffered more pain during the first 24 hours. From the third post-operative day onward, patients who underwent mini-thoracotomy suffered less pain. This is an important finding that may be explained by the fact that mobilization of patients with a mini-thoracotomy is rather painless as compared with full median sternotomy, in which the patient mobility causes less bony friction [18]. In our study, the total hospital stay was comparable in the two groups; the range of hospital stay in group "A" was 5-8 days with a mean of $6.6 \pm 0.9$ days, while in group "B" the range was 7-11 days with a mean of $8.8 \pm 0.8$ days. This shows that the total hospital stay in the minimally invasive group was less than sternotomy group, and this difference has a highly statistical significance. All the studies reported that hospital stay is significantly less in patients with minimal invasive approach than those with full sternotomy.

In 2014 Donald D. Glower, et al. reported a mean hospital stay of $6 \pm 4$ days in the mini-thoracotomy group, while a mean of $8 \pm$ 8 days in sternotomy group. It's lower in minimal invasive group. Mattiaglauber, et al. reported that the hospital stay ranged from 4-6 with a median of 5 days in the mini-thoracotomy group, while it was 5-7 days with a median of 6 days in sternotomy group. It's nearly equal in both groups [15,17]. Mauro Del Giglio, et al. mentioned thatthe hospital stay ranged from 6-8 with a median of 7 days. This method has definitive advantages, a reproducible, safe, and effective procedure. This will provide a clinical benefit to our patients, as well as advance our specialty $[16,19]$. Adoption rates are low due to complacency with conventional sternotomy techniques and the rapidly changing health care environment. This should not deter cardiac surgeons from providing advanced minimally invasive techniques to our patients. Whether an AVR is performed via a full sternotomy or a mini-thoracotomy, the size of the aortic annulus does not change. The surgeon needs to become comfortable working in a smaller space and become proficient with the use of long shafted instruments. Developing additional techniques and maneuvers within ones comfort zone will provide the necessary exposure. The devoted surgeon interested in developing a minimally invasive program needs to experience live case demonstrations, review videos of the procedure, read technical manuscripts, consider being proctored and finally begin the journey!

\section{Conclusion and Recommendations}

Right mini-thoracotomy AVR represents a shift in the approach to aortic valve surgery. We can conclude that minimal invasive AVR can be performed safely and effectively with very few peri-operative complications. The early outcomes in these 
patients are acceptable with less blood loss, less ICU stay and hospital stay, less incidences of major complications such as pain, wound infection and mediastinitis and with better post-operative pulmonary functions.

We recommend mandatory usage of intra-operative TEE allowing detection of most importantly air bubbling, paravalvular leakage. Furthermore we recommend adopting minimally invasive as safe and alternative approach in aortic valve surgery by mini-thoracotomy in patients with aortic valve disease requiring surgery as can as possible due to its better surgical outcome, better patient benefit, satisfaction and lower overall cost effectiveness.

\section{Acknowledgement}

The authors would like to acknowledge all the medical and nursing staff who has contributed to the data collection. Also we would like to thank cardiac surgeons and cardiologists who contributed in patients' management and we would like to thanks the research center's staff for their contribution to this research and for their assistance with revising, and their contribution to conception, design and processing of this project.

\section{References}

1. Pragnesh Joshi, Chirag Doshi, Mahesh Vinchurkar, Rajesh Thosani, Prashant Sagar, Vijay Mahajan. Minimally Invasive Combined Aortic and Mitral Valve Replacement. Heart, Lung and Circulation. 2011;20(4):231-233.

2. Cohn LH. Minimally invasive aortic valve surgery: technical considerations and results with the parasternal approach. J Card Surg. 1998;13(4):302-305.

3. Cosgrove DM, Sabik JF, Navia JL. Minimally invasive valve operations. Ann Thorac Surg. 1998;65(6):1535-1538.

4. Navia JL, Cosgrove DM. Minimally invasive mitral valve operations. Ann Thorac Surg. 1996;62(5):1542-1544.

5. Lawrence H Cohn. Cardiac surgery in adult book. $4^{\text {th }}$ edition. 2012.

6. Plass A, Scheffel H, Alkadhi H, Kaufmann P, Genoni M, Falk V, et al. Aortic valve replacement through a minimally invasive approach: preoperative planning, surgical technique, and outcome. Ann Thorac Surg. 2009;88(6):1851-1856. doi: 10.1016/j.athoracsur.2009.08.015

7. Gilmanov D, Bevilacqua S, Murzi M, Cerillo AG, Gasbarri T, Kallushi E et al. Minimally invasive and conventional aortic valve replacement: $A$ propensity score analysis. Ann Thorac Surg. 2013;96(3):837-843. doi: 10.1016/j.athoracsur.2013.04.102
8. Crawford FA, Kratz JM. Thoracic incisions. In Sabiston DC and Spencer FC (editors): Surgery of the Chest. sixth edition. W.B. Saunders Company; 1995.

9. Gilmanov D, Solinas M, Farneti PA, Cerillo AG, Kallushi E, Santarelli F, et al. Minimally invasive aortic valve replacement: 12 -year single center experience. Ann Cardiothorac Surg. 2015;4(2):160-169. doi: 10.3978/j. issn.2225-319X.2014.12.05

10.Inderbitzi RG, Schmid RA, Melfi FM, Casula RP. Minimally Invasive Thoracic and Cardiac Surgery Textbook and Atlas. Ch: 42 . Springer Berlin Heidelberg; 2012. p. 451-465.

11.Lehmann S, Merk DR, Etz CD, Seeburger J, Schroeter T, Oberbach A, et al. Minimally invasive aortic valve replacement: the Leipzig experience. Ann Cardiothorac Surg. 2015;4(1):49-56. doi: 10.3978/j.issn.2225319X.2014.11.03

12.Liu J, Sidiropoulos A, Konertz W. Minimally invasive aortic valve replacement (AVR) compared to standard AVR. Eur J Cardiothorac Surg. 1999.

13.Dogan S, Dzemali O, Wimmer-Greinecker G, Derra P, Doss M, Khan MF, et al. Minimally invasive versus conventional aortic valve replacement: a prospective randomized trial. J Heart Valve Dis. 12(1):76-80.

14.Brinkman WT, Hoffman W, Dewey TM, Culica D, Prince SL, Herbert MA, et al. Aortic Valve Replacement Surgery: Comparison of Outcomes in Matched sternotomy and PORT ACCESS Groups. Ann Thorac Surg. 2010;90(1):131-135. doi: 10.1016/j.athoracsur.2010.03.055

15.Glower DD, Desai BS, Hughes GC, Milano CA, Gaca JG. Aortic Valve Replacement via Right minithoracotomy versus median sternotomy, a Propensity Score Analysis. Innovations (Phila). 2014;9(2):75-81. doi: 10.1097/IMI.0000000000000062

16.Mauro Del Giglio, Simone Calvi, Marco Panzavolta, Marco Paris, Marco Pagliaro, Mauro Lamarra, et al. Aortic Valve Replacement through a Right Mini-Thoracotomy: Single Center Experience and Literature Review. 2015.

17.Glauber M, Ferrarini M, Miceli A. Minimally invasive aortic valve surgery: state of the art and future direction. Ann Cardiothorac Surg. 2015;4(1):26-32. doi: 10.3978/j.issn.2225-319X.2015.01.01

18.Aris A, Camara ML, Montiel J, Delgado LJ, Galan J, Litvan H. Mininsternotomy Versus Median Sternotomy for Aortic Valve Replacement: A prospecive Ranomized Study. Ann Thorac Surg. 1999;67(6):1583-1587.

19.Alassal MA, Ibrahim BM, Elsadeck N. Impact of aortic prosthesispatient mismatch on left ventricular mass regression. Asian Cardiovasc Thorac Ann. 2014;22(5):546-550. doi: 10.1177/0218492313500499 